\title{
Editorial
}

\section{Special issue based on the INFORMS Conference, June 2007, Barcelona, Spain}

\author{
Journal of Revenue and Pricing Management (2009) 8, 1-2. doi:10.1057/rpm.2008.59
}

This special issue showcases papers that were presented at the 7th Annual INFORMS Revenue Management and Pricing Section Conference that took place in Barcelona on June 28-29. Special thanks to Professor Kalyan Talluri and the organising committee of the conference for their hard work and efforts to make this meeting a great success. Attendees included academics and practitioners from around the world. The meeting was highlighted by keynote addresses from distinguished speakers Bruno Matheu, Executive Vice President of Marketing and Network Management at Air France, and Professor Xavier Vives of the IESE Business School at ICREA-UPF. The INFORMS Revenue Management and Pricing Section hopes to build on this success to attract more European members. This issue represents a sample of the research that was presented at the conference. Below I briefly discuss these papers.

The issue starts with a paper entitled 'Separable Approximations for Joint Capacity Control and Overbooking Decisions in Network Revenue Management' by Alexander Erdelyi and Huseyin Topaloglu. This paper tackles a network revenue management problem with capacity control and overbooking decisions. The authors apply an iterative simulation-based scheme to build separable approximations of the penalty cost of denying boarding and decompose the network revenue management problem by itineraries. The profits obtained by their model outperform deterministic heuristics by 3 per cent on average and by up to 13 per cent.
The second paper in the issue is entitled 'Optimal Bidding in On-line Auctions' by Dimitris Bertsimas, Jeffrey Hawkins and Georgia Perakis. The authors develop algorithms for optimally bidding for a single item in an online auction as well as for multiple items in multiple simultaneous or overlapping on-line auctions. Using real data from Ebay, they show that their dynamic policy outperforms widely used static heuristics, and propose a fast and effective heuristic for the multiple auctions problem using an integer programming framework. This method may also be effective in other settings with high-dimensional dynamic programming problems.

The third paper is entitled 'Dynamic pricing when customers strategically time their purchase: asymptotic optimality of a two price policy' by Matulya Bansal and Costis Maglaras. The authors consider the dynamic pricing problem faced by a monopolist in presence of strategic customers with varying valuations and risk preferences. They formulate the problem using a static mechanism design model. The authors establish structural properties of the optimal solution, and solve several special cases. In the low risk-aversion case, they use an asymptotic analysis to show that the widely used 'two-price point' strategy is near-optimal.

The next paper is entitled 'Multi-Product Pricing via Robust Optimization' by Aurelie Thiele. The author proposes an approach to model demand uncertainty in pricing problems with capacitated resources that uses range forecasts for various product lines, and bounds on the amount of resources that reflect the risk 
preferences of decision makers. She analyses the impact of uncertainty and risk aversion on the optimal strategy. She also provides an explicit characterisation of the impact of the system parameters on the optimal strategy when the price response function is linear as well as in cases of low uncertainty.

These are only a few examples of the exciting research that was presented at 7 th Annual INFORMS Revenue Management and Pricing Section Conference in Barcelona, Spain. In addition to the papers presented at the conference, this issue also contains another research paper, a practice paper, a book review and a futures paper.

The research paper is entitled 'Optimal pricing ladders for the sale of airline tickets' by Christine Currie and Daniel Simpson. The authors consider the factors that affect the discrete set of prices that can be charged for seats - also known as pricing ladders - for one-way economy tickets. They assume that the minimum and maximum fares are fixed and seek to maximise revenue by setting the spacing of the intermediate fares. They consider a single flight with perfect market segmentation and imperfect market segmentation and multiple flights on one route.

The practice paper is entitled 'Decision Support Model based on Risk-Return Tradeoff for Examining Viability of a Business Venture' by Sameer Kumar, Thomas Ressler and Mark Ahrens. The authors propose an integrated decision-support model for risk and revenue management that uses a four-step methodology. By performing subjective evaluations of revenue and risk options, this model enables the selection of an optimal product configuration for a new business venture.

In addition, this issue includes a detailed and enticing book review by Dax Cross of 'Money Ball', and a futures paper entitled 'Changing Revenue Management and Marketing Using a New Customer Lifecycle Management System' by Steven Pinchuk. The futures paper discusses a new differentiated dynamic market segmentation approach that can be used in revenue management and in marketing programs. The segmentation is focused on the life cycles of individual customers and leverages detailed data on customer behaviour and interaction.

I hope that you will enjoy this issue and share my excitement about the terrific new research and application of pricing and revenue management exhibited in these high-quality papers.

Soulaymane Kachani

Department of Industrial Engineering \& Operations Research, Columbia University,

NY, USA

E-mail:kachani@ieor.columbia.edu 